\title{
1 UeVO MODELO DE INNOVACIÓN PARA EL CONTEXTO ORGANIZACIONAL: UNA RELACIÓN ENTRE EL EMPRENDIMIENTO CORPORATIVO Y LAS PARTES INTERESADAS *
}

\author{
Novel innovation model for the organizational \\ context: A relationship between corporate \\ entrepreneurship and stakeholders
}

ID Pilar Valencia-DeLara*

ID Manuela Escobar-Sierra* ${ }^{\star \star \star}$

ID Felipe Calderón-Valencia ${ }^{* \star *}$

\footnotetext{
"El articulo hace parte de los resultados del proyecto de investigación titulado: “Emprendimientos corporativos socialmente responsables propuestos por la academia, un análisis desde los valores culturales", Financiado por la Universidad de Medellín, registrado en el CvLac y en el GrupLac del grupo de investigación "Jurídicas" - de la Facultad de Derecho- y "CITIE Ciencia, tecnología, innovación y emprendimiento" -de la Facultad de Ciencias Económicas y Administrativas- de la misma IES.

" Universidad de Medellín, Facultad de Ciencias Económicas y Administrativas, grupo de investigación CITIE, Medellín - Colombia, E-mail: mivirpi15@gmail.com; mpvalencia@udem.edu.co

*** Universidad de Medellín, Facultad de Ciencias Económicas y Administrativas, grupo de investigación CITIE, Medellín - Colombia, E-mail: manuelaescobar@gmail.com; maescobar@udem.edu.co

**** Universidad de Medellín, Facultad de Derecho, grupo de Investigación Jurídicas, Medellín - Colombia. E-mail: felipecalderonvalencia@gmail.com; fcalderon@udem.edu.co
}

Fecha de recepción: 8 de febrero del 2018

Fecha de aceptación: 15 de mayo del 2018

\section{Cómo Referenciar / How to cite}

Valencia-DeLara, P., Escobar-Sierra, M. \& Calderón-Valencia, F. (2018). Nuevo modelo de innovación para el contexto organizacional: una relación entre el emprendimiento corporativo y las partes interesadas. trilogía Ciencia Tecnología Sociedad, 10(19), 99-114. 
Resumen: el propósito del presente estudio es proponer un nuevo modelo de innovación para el contexto organizacional, ante la falta de consenso en torno al tema y la ausencia de un modelo de innovación generalizable e integrador. Para lograr este objetivo se desarrolla un análisis bibliométrico mediante el software VOSviewer ${ }^{\circledR}$, que permite identificar conceptos recurrentes a los modelos de innovación, entre los que se resaltan la competitividad, las organizaciones, las culturas y los sistemas. Posteriormente, estos hallazgos pasan a ser contrastados con en el caso de estudio colombiano, que sirve como fundamento para proponer un nuevo modelo de innovación para el contexto organizacional, desde su relación con las partes interesadas y el emprendimiento corporativo, en donde el punto de partida y de llegada son las mismas organizaciones y las partes interesadas quienes desarrollan sus procesos de innovación siguiendo alguno de los modelos referenciados en la literatura. Finalmente, se resalta la importancia de incluir nuevos actores que propicien la interdisciplinariedad de los estudios sobre innovación, la medición de sus resultados, la aplicación del modelo propuesto en contexto empíricos para propiciar su generalización, la definición de políticas e instrumentos públicos conexos que permitan ampliar al alcance del modelo, entre otros.

Palabras clave: organización; modelos de innovación; emprendimiento corporativo; partes interesadas; análisis bibliométrico.

Abstract: This study proposes a new innovation model for the organizational context that addresses the lack of consensus on the subject and the absence of a generalizable and comprehensive alternative. For that purpose, a bibliometric analysis was carried out using VOSviewer® software to find frequent terms in innovation models. Among the results, 'competitiveness', 'organizations', 'cultures', and 'systems' stood out. Subsequently, these findings were compared with the Colombian study case to present a new innovation model for the organizational context. T he model is based on the relationships between stakeholders and corporate entrepreneurship, and its starting point and focus are organizations and stakeholders who adopt one of the models of the literature to conduct their innovation processes. Finally, among other aspects, this work highlights the importance of including new stakeholders who provide innovation studies with interdisciplinarity, the measurement of results, the application of the proposed model in empirical contexts to enable generalization, and the definition of related public policies and tools that enable to expand the scope of the model.

Keywords: organization, innovation models, corporate entrepreneurship, stakeholders, bibliometric analysis. 


\section{INTRODUCCIÓN}

En el contexto competitivo actual, el creciente interés por la innovación y su supuesta relación con el crecimiento económico (Valencia-De-Lara y Patlán-Peréz, 2011a, 2011b), han favorecido los estudios en torno a la innovación (Forrest, 1991), entendida esta como una serie de actividades, un proceso ordenado (Godin, 2015), una secuencia de acontecimientos o esquematizaciones de historias (Van-de-Ven, Polley, Garud, y Venkataraman, 2008) orientados hacia la obtención de una ventaja competitiva temporal o absoluta (Rammer, 2006) y conceptualizadas a través de los llamados modelos de innovación. Modelos de innovación que han tratado de ser caracterizados por diversos autores, discusión en donde algunos plantean no haber llegado hasta la fecha a un consenso general sobre el tema, evidente a través de la ausencia de un modelo generalizable e integrador (Cooper, 1983; Godin, 2015; Hobday, 2005), mientras que otros los clasifican superficialmente en modelos corporativos propios de organizaciones maduras y en modelos de emprendimiento típicos de compañías nacientes (Freeman y Engel, 2007).

En este contexto, en donde la organización madura o incipiente es la base de los llamados modelos de innovación, se presenta una revisión histórica a través de la referenciación de varias propuestas y la realización de un análisis bibliométrico aplicado a la información disponible sobre modelos de innovación en las bases de datos indexadas por WOS (Web of Science), KCI - Korean Journal Database, Russian Science Citation Index, SciELO Citation Index y Scopus; posteriormente se selecciona el contexto colombiano como caso de estudio, para pasar a caracterizar el rol de las organizaciones a partir de conocimiento empírico y teórico disponible; una vez revisado este caso se propone un nuevo modelo de innovación para el contexto organizacional a partir del análisis de la información recolectada, para finalmente discutir los resultados obtenidos y concluir sobre los hallazgos.

\section{EVOLUCIÓN HISTÓRICA DE LOS MODELOS DE INNOVACION}

Una vez introducido el tema de discusión, se presenta en la Tabla 1 un recorrido histórico por los modelos de innovación propuestos por diferentes autores, en donde se incluyen algunos de los llamados modelos tempranos o "early models", hasta revisiones posteriores de modelos referenciados en la literatura desde los años 60 (Godin, 2015).

En línea con el recorrido histórico referenciado, a continuación, se realiza una revisión de la literatura sobre modelos de innovación indexada en la colección principal de WOS (Web of Science), KCI - Korean Journal Database, Russian Science Citation Index, SciELO Citation Index y Scopus, a partir del año 1972, con el fin de verificar el estado reciente de la discusión. Esta revisión muestra que 2.470 trabajos han sido orientados al estudio de los modelos de innovación como tema indexado en las bases de datos, cifra a la que se llega después de revisar las diferentes ecuaciones de búsqueda en inglés, idioma con mayor índice de publicación (Englander, 2009), tal como se presentan en la Tabla 2. 
NUEVO MODELO DE INNOVACIÓN PARA EL CONTEXTO ORGANIZACIONAL: UNA

RELACIÓN ENTRE EL EMPRENDIMIENTO CORPORATIVO Y LAS PARTES INTERESADAS

Tabla 1. Modelos de innovación propuestos

\begin{tabular}{|c|c|}
\hline Autor & Propuesta \\
\hline Chin, 1961) & Modelo para el desarrollo, de sistemas y para el cambio \\
\hline Clark, 1968) & $\begin{array}{l}\text { Modelo de crecimiento orgánico, de diferenciación, de difusión y del proceso } \\
\text { combinado }\end{array}$ \\
\hline Allen, 1967a, 1967b) & Modelo de izquierda a derecha y el modelo "wheel, hub and axle" \\
\hline Schon, 1967) & Modelo racional y no racional \\
\hline Havelock, 1970) & Modelo simple reflex y el racional de resolución de problemas \\
\hline Robertson, 1971) & Modelo de adopción, de jerarquía a efectos y AIDA (attention, interest, desire, action) \\
\hline $\begin{array}{l}\text { Langrish, Gibbons, Evans, y } \\
\text { Jevons, 1972) }\end{array}$ & Modelo de descubrimiento - push y de demanda - pull. \\
\hline Crane, 1972) & Modelo acumulativo (secuencial), aleatorio y de la curva logística \\
\hline $\begin{array}{l}\text { Zaltman, Duncan, y } \\
\text { Holbeck, 1973) }\end{array}$ & Modelo orientado al individuo y modelo orientado a la organización \\
\hline Mulkay, 1975) & Modelo de apertura, cerrado y ramificado \\
\hline Kelly y Kranzberg, 1978 & Modelo de fases de proceso y de flujo \\
\hline Von Hippel, 1978 & Modelo activo de producción y activo de clientes \\
\hline Schmidt-Tiedemann, 1982 & Modelo de tubería, sistemático, combinado y de concomitancia \\
\hline Tornatzky et al., 1983 & Modelo centrado en la tecnología y modelo de tecnología centrado en el usuario \\
\hline Pinch y Bijker, 1984 & Modelo lineal y multidimensional \\
\hline Saren, 1984 & $\begin{array}{l}\text { Modelo departamental, de actividades por etapas, de decisión por etapas, del proceso } \\
\text { de conversión y de respuesta }\end{array}$ \\
\hline Kline y Rosenberg, 1986 & Modelo lineal y de cadena \\
\hline $\begin{array}{l}\text { Coombs, Saviotti, y Walsh, } \\
1987\end{array}$ & Modelo lineal, evolutivo y epidémico \\
\hline Van de Ven, 1989 & $\begin{array}{l}\text { Modelo de desarrollo del grupo, del proceso de decisión, de planificación } \\
\text { organizacional, de procesos de innovación y de cambio y desarrollo organizacional }\end{array}$ \\
\hline Forrest, 1991 & $\begin{array}{l}\text { Modelo por etapas, de conversión, de tecnología push / mercado- pull, integrado y de } \\
\text { decisión }\end{array}$ \\
\hline Ziman, 1991 & Modelo lineal, cíclico y de red neuronal \\
\hline Newby, 1992 & Modelo lineal e interactivo \\
\hline Rothwell, 1992 & $\begin{array}{l}\text { Modelo de tecnología - push, de mercado - pull, de acoplamiento, integrado, de } \\
\text { integración estratégica y de redes }\end{array}$ \\
\hline Chris Freeman, 1996) & Modelo lineal y sistemático \\
\hline Tait y Williams, 1999) & Modelo lineal y modelo lineal-plus \\
\hline Marinova y Phillimore, 2003) & $\begin{array}{l}\text { Modelo de la caja negra, lineal, interactivo, de sistemas, evolutivo y de medios } \\
\text { innovadores }\end{array}$ \\
\hline Hargrave y Van De, 2006) & $\begin{array}{l}\text { Modelo de diseño institucional, de adaptación institucional, de difusión institucional } \\
\text { y de acción colectiva }\end{array}$ \\
\hline $\begin{array}{l}\text { Caraça, Lundvall, y } \\
\text { Mendonça, 2009) }\end{array}$ & Modelo lineal, chain-linked y de aprendizaje interactivo de varios canales \\
\hline
\end{tabular}

Fuente: elaboración autores. 
Tabla 2. Criterios de búsqueda y número de publicaciones en WOS, KCI, RSCI, SciELO y Scopus

\begin{tabular}{|l|l|}
\hline \multicolumn{1}{|c|}{$\begin{array}{c}\text { Ecuación de búsqueda en los títulos de las } \\
\text { publicaciones }\end{array}$} & \multicolumn{1}{c|}{ Número de registros } \\
\hline \multirow{2}{*}{ "Innovation models" } & WOS, KCI, RSCI, SciELO - 174 (desde 2001) \\
\cline { 2 - 2 } "Models of innovation" & Scopus - 1.478 (desde 1972) \\
\hline \multirow{2}{*}{ "MOS, KCI, RSCI, SciELO - 101 (desde 2001) } \\
\cline { 2 - 2 } & Scopus - 717 (desde 1975) \\
\hline
\end{tabular}

Fuente: elaboración autores.

Una vez se extraen los datos sobre autor, título, fuente y resumen, se pasa a procesar estos datos a través del software VOSviewer ${ }^{\circledR}$ versión 1.6.4.; con esta información se realiza un análisis bibliométrico (aplicación de métodos matemáticos y estadísticos a libros, artículos y otros medios de comunicación) para medir cantidad, desempeño e incluso algunos indicadores estructurales que determinan las conexiones entre publicaciones, autores y áreas de investigación que permiten vislumbrar el estado actual de la discusión sobre el tema a partir de índices (resultados numéricos) y mapas (representaciones gráficas) (Durieux y Gevenois, 2010; Romo-Jiménez, Valencia-DeLara, y Escobar-Sierra, 2017). A continuación, se presenta en la Figura 1 el mapa de conocimiento para los modelos de innovación clasificado por segmento de análisis y concurrencia de los términos en las bases de datos seleccionadas.

Figura 1. Mapa del conocimiento para los modelos de innovación

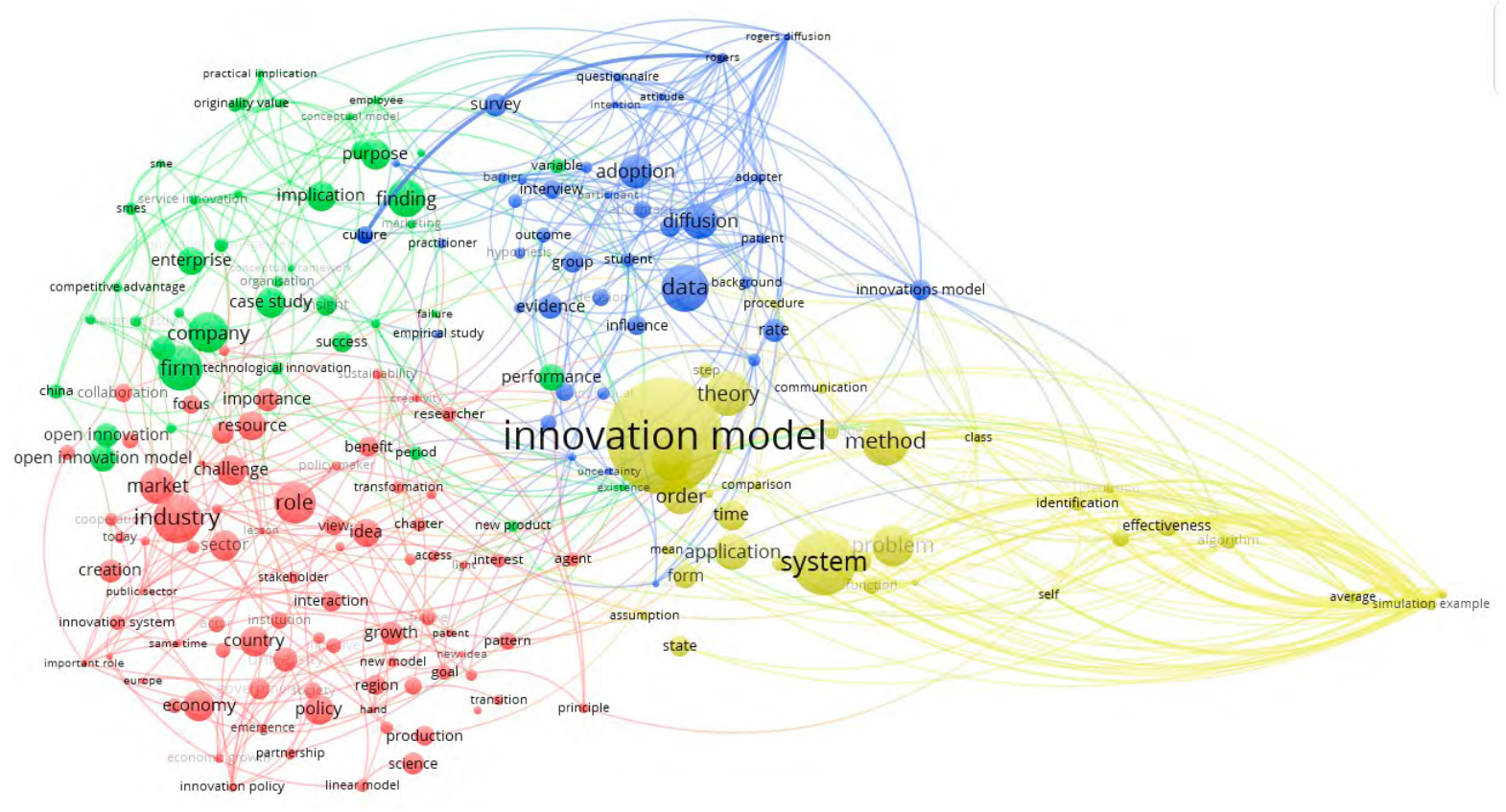

Fuente: elaboración de autores a través del software VOSviewer®. 
Nuevo MOdelo DE INNOVACIÓN PARA EL CONTEXTO ORGANIZACIONAL: UNA RELACIÓN ENTRE EL EMPRENDIMIENTO CORPORATIVO Y LAS PARTES INTERESADAS

Para resaltar el volumen creciente de investigaciones alrededor del tema en los últimos siete años, tal como se presenta en la Figura 2, y la concurrencia (concurso simultáneo de varias circunstancias (Real Academia Española, 2017), en este caso conceptos que se repiten más de 30 veces) de estudios sobre los modelos de innovación. Para el análisis de concurrencia, presentado en la Figura 1 con el mapa de conocimiento para los modelos de innovación, se destacan cuatro segmentos de análisis -diferenciados por color: rojo, verde, azul y amarillo- claramente definidos para los que los términos que presentan mayor concurrencia, así: el primer (1) segmento de análisis, corresponde a términos como: industria, rol, mercado, economía, país, sector y emprendimiento; el segundo (2) segmento de análisis, lo componen términos que hacen referencia a: firma, compañía, innovación abierta, éxito y gerencia; el tercer (3) segmento de análisis comprende términos orientados a: difusión, adopción, educación, decisión, programa y cultura; mientras que el cuarto (4) segmento de análisis, resalta términos como: sistema, método, teoría, problema, aplicación, tiempo, Estado y efectividad.

Figura 2. Número de publicaciones anuales sobre modelos de innovación a partir del 2000

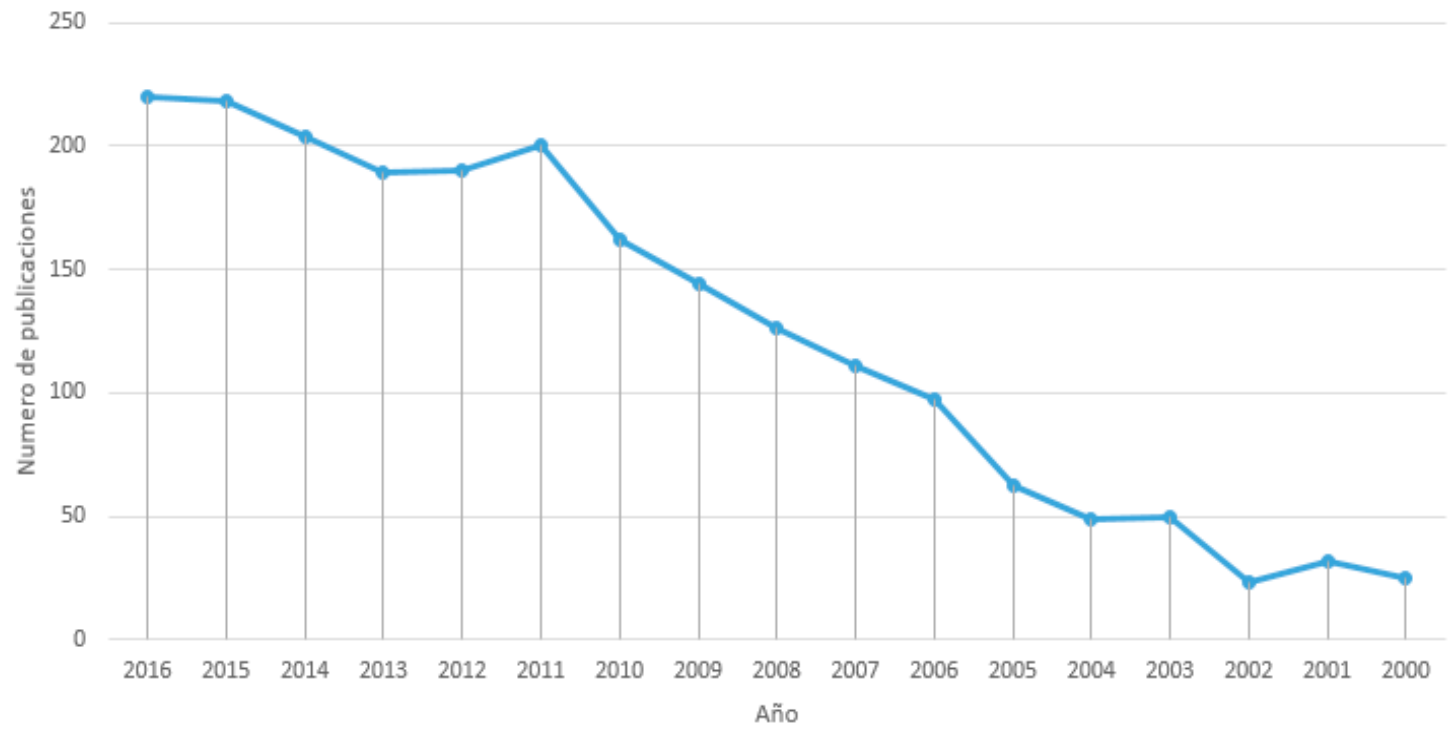

Fuente: elaboración autores.

En línea con los resultados obtenidos con el software VOSviewer® se verifica el reciente planteamiento de otros autores, que se han esforzado en caracterizar los desafíos que enfrentan los modelos de innovación, hasta proponer la necesidad de incluir: asuntos ambientales y ecológicos en el estudio sobre el tema (Marinova y Phillimore, 2003), dimensiones sociales que consideren las diferentes partes interesadas (Escobar-Sierra, Lara-Valencia, \& Valencia-DeLara, 2017; Quesada-Vázquez \& Rodríguez-Cohard, 2015), etapas preliminares del proceso innovador como la ideación y el diseño
(Hennala, Parjanen, y Uotila, 2011; Mike Hobday, Boddington, y Grantham, 2012), nuevos actores que propicien la interdisciplinariedad de su análisis (Hennala et al., 2011; Quesada-Vázquez y Rodríguez-Cohard, 2015), diferentes modelos para integrarlos durante la implementación (Friederichsen, Minh, Neef, y Hoffmann, 2013), políticas e instrumentos públicos conexos (Gokhberg y Kuznetsova, 2011; Wojan, 2017), $\mathrm{y}$ conceptos cercanos como el emprendimiento corporativo (Corbett, Covin, O'Connor, y Tucci, 2013; Hashimoto y Nassif, 2014). 
Una vez caracterizado el estatus actual de la discusión teórica en torno a los modelos de innovación, es oportuno contrastar los hallazgos teóricos revisados con el contexto empírico colombiano, para discutirlos. La selección de este caso de estudio se hace luego de reconocer los logros y desafíos que enfrenta la innovación que se desarrolla en el contexto organizacional del país, logros entre los que se resalta el ascenso en el escalafón global de innovación que presentó para el 2017 (The Global Innovation Index, 2017) y el beneficio que esto representa para las empresas nacionales, y retos entre los que se subraya el preocupante índice de desempleo nacional que para Julio de 2017 se acercó nuevamente a los dos dígitos al ubicarse en 9,7 \% (DANE, 2017) y tiende a aumentar ante la explosiva llegada de venezolanos al país (Migración Colombia, 2017) y la desmovilización de algunos actores armados tras la firma del acuerdo de paz (Gobierno Nacional y FARC-EP, 2016).

Estas situaciones convocan a las compañías colombianas a actuar (ACDIVOCA, 2017) como actores principales del desarrollo económico, dinamizadoras del mercado y generadoras de empleo (OCDE, 2014; Valencia-De-Lara y Patlán Peréz, 2011a, 2011b). En este sentido, se propone en el siguiente numeral una introducción al contexto seleccionado a través de algunos datos secundarios, para terminar con la revisión y análisis del conocimiento empírico y teórico disponible en torno al tema, que permitan caracterizar los desafíos del modelo de innovación a nivel organizacional.

\section{DESAFÍOS QUE ENFRENTA LA INNOVACIÓN EN EL CONTEXTO ORGANIZACIONAL COLOMBIANO}

Para hablar de innovación en el contexto organizacional colombiano es pertinente comprender el contexto innovador del país, Colombia adoptó desde 1990 la propuesta teórica de autores como C. Freeman (1991), Lundvall (2010) y Nelson (1993, 2000), con su Sistema
Nacional de Innovación (modelo previamente referenciado por autores como Chin (1961), Chris Freeman (1996)y Marinova y Phillimore (2003)). Inicialmente el modelo de innovación adoptado fue denominado como Sistema Nacional de Ciencia y Tecnología (SNCYT) configurado en la Ley 29 de 1990 (Congreso de la República de Colombia, 1990), hasta llamarlo Sistema Nacional de Innovación colombiano (SNCTI) en la Ley 1286 de 2009 (Congreso de la República de Colombia, 2009), momento en donde ambos sistemas fueron fusionados. Luego de 17 años de aplicación de la política pública adoptada, existen algunos avances, que reconocen reportes como el índice global de innovación, donde Colombia ha subido 25 puestos en los últimos siete años (The Global Innovation Index, 2017), mientras que otros asuntos como la inversión en ciencia (ACCEFYN, 2017) permanecen pendientes.

En este sentido, se resaltan a continuación algunos asuntos pendientes que involucran a las compañías y que merecen especial atención para comprender el rol protagónico de las organizaciones en el ecosistema innovador y los desafíos que enfrentan, entre ellas se alerta sobre: el salto que deben dar los empresarios para pasar de las ideas a los negocios reales (ANDI, 2017); el comportamiento de la inversión en actividades de ciencia, tecnología e innovación en el país, en donde se evidencia que las empresas han incrementado sus aportes en los últimos 10 años, mientras las entidades públicas los han reducido (Consejo privado de competitividad, 2016); la preferencia de las fuentes internas de ideación a nivel empresarial (DANE, 2014, 2015); la tendencia creciente de la discontinuidad en la actividad empresarial, atribuida principalmente a la baja rentabilidad financiera de los negocios (GEM, 2014) y relacionada con la tasa de mortandad a nivel empresarial; la incertidumbre ante una reforma tributaria (ANDI, 2016); la ausencia de políticas que consoliden una cultura de innovación empresarial y emprendimiento dinámico (Fedesarrollo, 2014); la pobre generación de conocimiento a nivel 
Nuevo MOdelo DE INNOVACIÓN PARA EL CONTEXTO ORGANIZACIONAL: UNA RELACIÓN ENTRE EL EMPRENDIMIENTO CORPORATIVO Y LAS PARTES INTERESADAS

empresarial, en donde las empresas innovadoras en sentido estricto no superan el $29 \%$ (DNP, Observatorio colombiano de ciencia y tecnología, \& C230 Consultores, 2015); la importancia de situar al sector empresarial en el centro del Sistema Nacional de Innovación colombiano (OCDE, 2014); y el alcance de los emprendedores de la nación que se ha caracterizado por ser local (Endeavor Colombia, 2016).

En línea con estas alertas, necesidades, falencias y hallazgos, algunos autores van más allá y se atreven a proponer alternativas organizacionales como: el crecimiento verde y el aprovechamiento de los recursos hídricos, los desafíos a gestionar por los empresarios (ANDI, 2015), la innovación abierta como forma de integración entre las partes interesadas en el ecosistema innovador (Accenture, 2016), la búsqueda de inversión privada para emprender que soporte la actual fuente de financiación de los nuevos empresarios del país, quienes se apalancan en recursos propios principalmente (GEM, 2014) y la innovación como sentido humano en las empresas como fuente de ventaja, entendiendo que el negocio de la innovación es sobre la gente y por tanto las organizaciones deben ser un lugar donde las personas se sientan inspiradas a ir a trabajar, y se sientan a gusto con lo realizado durante el día (Mead, 2016).

Entre tanto, algunos autores se atreven a plantear los desafíos que enfrenta la innovación del contexto organizacional a nivel teórico en Colombia, entre ellos Gómez, Martínez, y Arzuza (2006) quienes al revisar las normas existentes sobre creación de empresas (proceso del SNCTI) las califican como dispersas, poco claras y generales; QuinteroCampos (2010) por su parte, alerta sobre la necesidad de medir los resultados de la innovación, así como la importancia de precisar las necesidades relativas a las políticas concernientes a cada uno de los agentes; Robledo (2007) quién analiza el rol de los grupos de investigación universitarios dentro del sistema y su disposición para integrarse con las diferentes partes interesadas en pro de articular sus investigaciones con los procesos de innovación; Monroy Varela (2006), entre tanto, estudia el bajo nivel de interacción entre los agentes del SNCTI y propone nuevas políticas y estrategias que permitan replantear el funcionamiento del sistema para tratarlo como una organización social abierta que cuente, entre otros, con personal capacitado y recursos económicos; y finalmente, López (2004) quién critica la falta de capacidad de consultoría especializada en investigación, desarrollo e innovación, la ausencia de estudios de prospectiva tecnológica, la falta de claridad en el manejo de la propiedad intelectual, y el pobre fortalecimiento de los procesos conducentes a alianzas tecnológicas nacionales e internacionales.

Para resaltar luego de este recorrido empírico y teórico en torno a la innovación en el contexto organizacional colombiano, los hallazgos previamente referenciados en el análisis bibliométrico, entre los que se relaciona la necesidad de incluir los siguientes aspectos: (1) asuntos ambientales y ecológicos en el estudio sobre el tema (Marinova y Phillimore, 2003), tal como lo sugiere la ANDI (2015) al plantear los desafíos de gestión de los empresarios; (2) la introducción de dimensiones sociales en donde se incluyan las diferentes partes interesadas (Estado, socios, colaboradores, proveedores, comunidad, academia) (Gokhberg y Kuznetsova, 2011; Quesada-Vázquez y Rodríguez-Cohard, 2015) en su ejecución, entre las que se resaltan para el caso de estudio seleccionado (Colombia y sus organizaciones) alianzas para generar empleos (ACDIVOCA, 2017), apuestas por la innovación abierta (Accenture, 2016), la innovación como sentido humano en las empresas para vincular a sus empleados (Mead, 2016), participación de los grupos de investigación universitarios (Robledo, 2007), integración entre los agentes del ecosistema (Monroy-Varela, 2006), el sector empresarial en el centro del Sistema Nacional de Innovación colombiano (OCDE, 2014), alianzas tecnológicas nacionales e internacionales (López, 2004); y (3) políticas e instrumentos públicos conexos que 
permitan, entre otras, ampliar al alcance del modelo de innovación adoptado (Gokhberg y Kuznetsova, 2011; Wojan, 2017), tal como lo confirman en el contexto de estudio Monroy Varela (2006) al proponer un replanteamiento del SNCTI; Gómez et al. (2006) quienes critican las normas existentes sobre creación de empresas; Quintero-Campos (2010) cuando resalta la importancia de precisar las necesidades relativas a las políticas en cada uno de los agentes; Fedesarrollo (2014), al proponer sus 15 recomendaciones de política; y la ANDI (2016) al alertar sobre el impacto de la reforma tributaria.

\section{PROPUESTA DE UN NUEVO MODELO DE INNOVACIÓN EN EL CONTEXTO ORGANIZACIONAL}

En este sentido, se propone un nuevo modelo de innovación en el contexto organizacional para Colombia, donde se integren los hallazgos empíricos y teóricos previamente comentados, en aras de replantear el funcionamiento del actual
SNCTI, tal como lo sugiere Monroy-Varela (2006), partiendo del rol protagónico de las organizaciones empresariales (OCDE, 2014) y (Valencia-De-Lara y Patlán Peréz, 2011a, 2011b). Bajo esta premisa, se definen categorías críticas del estudio a nivel teórico y empírico, para pasar luego a integrarlas en el denominado nuevo modelo de innovación en el contexto organizacional. Entre estas categorías críticas del estudio se resalta la inclusión de asuntos ambientales (ANDI, 2015; Marinova y Phillimore, 2003), la integración entre agentes o partes interesadas (Accenture, 2016; ACDIVOCA, 2017; Gokhberg y Kuznetsova, 2011; Quesada-Vázquez y RodríguezCohard, 2015), la incorporación de conceptos cercanos como el emprendimiento corporativo (Corbett, Covin, O'Connor, y Tucci, 2013; Hashimoto y Nassif, 2014), y la selección o integración de modelos de innovación a la medida (Friederichsen et al., 2013; Hennala et al., 2011; Mike Hobday et al., 2012) para integrarlos en el modelo que se presenta a continuación en la Figura 3.

Figura 3. Nuevo modelo de innovación en el contexto organizacional

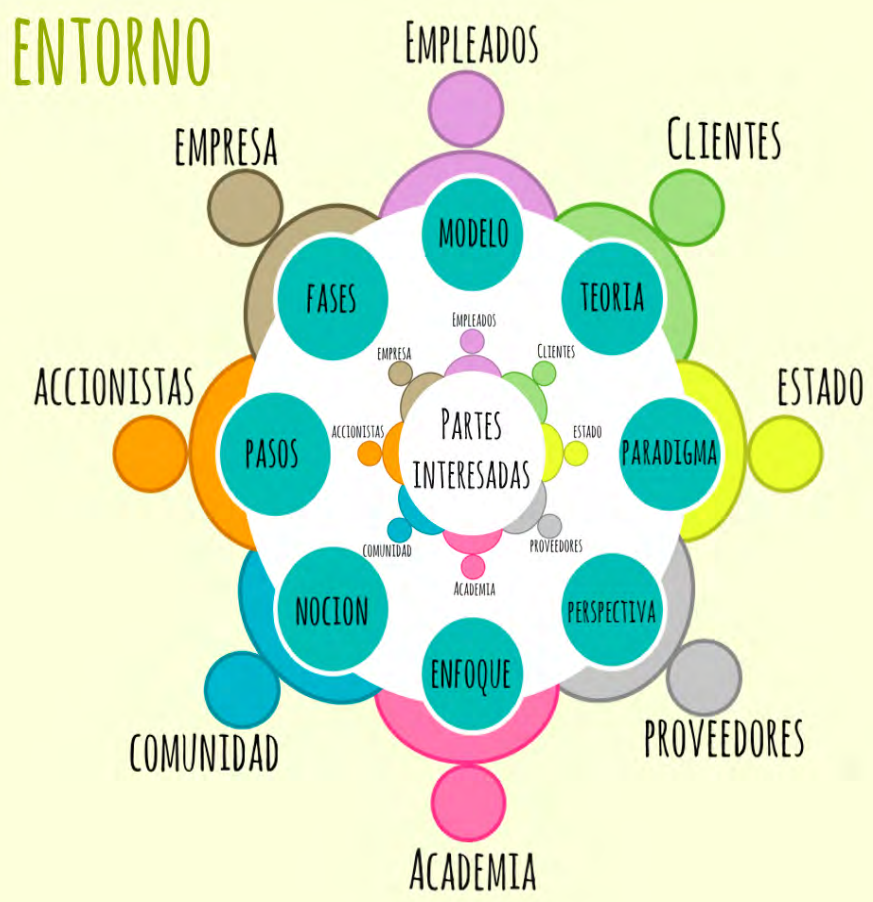

Fuente: elaboración autores. 
Nuevo MODElO DE INNOVACIÓN PARA EL CONTEXTO ORGANIZACIONAL: UNA RELACIÓN ENTRE EL EMPRENDIMIENTO CORPORATIVO Y LAS PARTES INTERESADAS

En esta propuesta el punto de partida y el punto de llegada para gestionar la innovación son los mismos stakeholders o partes interesadas, definidos en este caso como empresas, empleados, clientes, Estado, proveedores, academia, accionistas y comunidad, quienes en conjunto componen el ecosistema del Sistema Nacional de Innovación ( Freeman, 1991; Lundvall, 2010; Nelson, 1993, 2000).

Específicamente para el caso de las organizaciones, eje central de la discusión, se propone abordar los modelos de innovación en el interior de la compañía desde constructos afines como el emprendimiento corporativo (Guth y Ginsberg, 1990; Morris, Kuratko, y Covin, 2010), adaptando elementos propios de su conceptualización (Fang, 2013), a través de un sistema compuesto por antecedentes, procesos y salidas. Así los antecedentes se centran en el entorno, la estrategia y los factores organizacionales (Amaeshi, Nnodim, y Osuji, 2013; Kuratko, 2010; Zahra, 1986), mientras que los procesos son vinculados con la orientación emprendedora caracterizada por la innovación, la proactividad y la disposición al riesgo (Covin y Slevin, 1991; Morris, Kuratko, y Covin, 2010) y las salidas con su relación con el desempeño, entendido este como una medida del crecimiento en las ventas, la participación en el mercado, la rentabilidad, el desempeño y la satisfacción de las partes interesadas (Escobar-Sierra y Vera-Acevedo, 2016; Cao, Simsek, y Jansen, 2015).

Las situaciones descritas convierte a las organizaciones y en general a cada uno de los stakeholders o partes interesadas en terreno fértil sobre el que se desarrollan procesos de innovación a la medida que pueden ser llevados a cabo siguiendo alguno de los modelos de innovación, también llamados teorías, patrones, enfoques, esquemas, paradigmas, marcos, representaciones, perspectivas, nociones, hipótesis, figuras $\mathrm{y}$ diagramas referenciados en la literatura (Godin, 2015). Finalmente, es importante mencionar que tanto las organizaciones y en general los stakeholders o partes interesadas como el modelo seleccionado para la innovación, deben de estar enmarcados dentro de las necesidades de las diferentes partes interesadas (Freeman y Engel, 2007) como indicadores de desempeño.

\section{DISCUSIÓN CON OTROS AUTORES}

El nuevo modelo de innovación propuesto para el caso de estudio colombiano, atiende las sugerencias teóricas de ciertos autores, mientras omite las propuestas de otros. Entre las consideraciones teóricas que atiende se encuentra la inclusión de asuntos ambientales y ecológicos en el estudio sobre el tema (Marinova y Phillimore, 2003), la revisión de dimensiones sociales que incluyan las diferentes partes interesadas o stakeholders (Gokhberg y Kuznetsova, 2011; Monroy Varela, 2006; QuesadaVázquez y Rodríguez-Cohard, 2015), la atención a etapas tempranas del proceso innovador como la ideación y el diseño (Hennala et al., 2011; Mike Hobday et al., 2012), el análisis desde conceptos afines como el emprendimiento corporativo (Corbett, Covin, O'Connor, y Tucci, 2013; Hashimoto y Nassif, 2014) y la integración de diferentes modelos durante la implementación (Friederichsen et al., 2013).

Mientras que al referirse a las propuestas teóricas que ignora, se encuentra la propuesta de aquellos autores que proponen la inclusión de nuevos actores que propicien la interdisciplinariedad de su análisis (Hennala et al., 2011; Quesada-Vázquez y Rodríguez-Cohard, 2015), la definición de políticas e instrumentos públicos conexos que permitan, entre otras, ampliar el alcance del modelo de innovación adoptado (Gokhberg y Kuznetsova, 2011; Monroy Varela, 2006; Wojan, 2017), la medición de los resultados de la innovación, así como la precisión de las necesidades relativas a las políticas en cada uno de los agentes propuestos por Quintero-Campos (2010). 


\section{CONCLUSIONES Y RECOMENDACIONES}

En el nuevo modelo de innovación propuesto, el punto de partida para gestionar la innovación son las organizaciones y en general los mismos stakeholders o partes interesadas, definidos como las empresas, los empleados, los clientes, el Estado, los proveedores, la academia, los accionistas y la comunidad, quienes en conjunto componen el ecosistema del Sistema nacional de Innovación. En este contexto, cada uno de los actores citados, y en especial la organización, dinamizadora del mercado, debe gestionar su estrategia, los factores relacionados con el recurso humano y el medio en el que opera, para desarrollar procesos de innovación a la medida que bien pueden ser aplicados siguiendo alguno de los múltiples modelos referenciados en la literatura, garantizando que estén enmarcados dentro de las necesidades de las diferentes partes interesadas o stakeholders, como indicadores del desempeño.

Se resalta la pertinencia del recorrido histórico y el análisis bibliométrico sobre los modelos de innovación, para establecer los antecedentes de su estudio y sus actuales segmentos de análisis o categorías de investigación teórica, donde sobresale la falta de consenso en torno a la definición de un modelo de innovación generalizable, la explosión de su estudio en los últimos siete años y la presencia de cuatro segmentos de análisis que pueden sintetizarse en temas de competitividad, organizaciones, culturas y sistemas tal como lo sugiere el análisis bibliométrico desarrollado en el numeral 2. Hallazgos que posteriormente son triangulados con el caso colombiano donde a partir de la revisión de datos secundarios y conocimiento experto se verifican hallazgos previos, hasta desarrollar el nuevo modelo de innovación para el contexto organizacional y proyectar temas pendientes de investigación entre los que se relaciona la importancia de incluir nuevos actores que propicien la interdisciplinariedad de su análisis, la medición de los resultados de innovación, la definición de políticas e instrumentos públicos conexos que permitan ampliar al alcance del modelo de innovación adoptado, entre otros.

\section{REFERENCIAS}

ACCEFYN. (2017). Recorte del 41\% del presupuesto para ciencia genera indignación y protestas. Revista Dinero. Recuperado de http://www.dinero. $\mathrm{com} /$ pais / articulo/ cientificos-convocanplanton-por-presupuesto-para-ciencia-ytecnologia/ 249056

Accenture. (2016). Innovación abierta eleva el PIB entre 2 y $4 \%$ : Accenture. Medellín. Recuperado de http://www.elcolombiano.com/negocios/ innovacion-colaborativa-un-motor-que-elevael-pib-CC4532334

ACDIVOCA. (2017). Empresas firman Alianzas para la Reconciliación. Portafolio. Recuperado de http:/ / www.portafolio.co/negocios/ empresas/ empresas-firman-alianzas-para-lareconciliacion-508623

Allen, J. A. (1967a). Scientific innovation and industrial prosperity. Amsterdam: Elsevier Applied Science.

Allen, J. A. (1967b). Studies in innovation in the steel and chemical industries (Vol. 78). Ireland: Manchester University Press.

Amaeshi, K., Nnodim, P., \& Osuji, O. (2013). Corporate social responsibility, entrepreneurship, and innovation. New York: Routledge. https:/ / doi.org/10.4324/9780203081945

ANDI. (2015). Estrategia para una nueva industrialización. Bogotá. Recuperado de http: / / www.andi.com.co/ Asamblea/ Documents / Libro / Estrategia\%20 para $\% 20$ una\%20nueva\%20industrializacion.pdf

ANDI. (2016). Innovation Land 2016 comenzó con preocupación de los empresarios. Medellín. Recuperado de http://www.elcolombiano. com/negocios/innovacion/ colombia- 
Nuevo MOdelo DE INNOVACIÓN PARA EL CONTEXTO ORGANIZACIONAL: UNA

RELACIÓN ENTRE EL EMPRENDIMIENTO CORPORATIVO Y LAS PARTES INTERESADAS

empresas-comenzo-innovation-land-2016FA5424403

ANDI. (2017). Empresarios deben pasar de las ideas a negocios reales. El Colombiano. Recuperado de http://www.elcolombiano.com/negocios/ empresarios-colombianos-renuevan-planestrategico-AY7098046

Cao, Q., Simsek, Z., \& Jansen, J. J. P. (2015). CEO social capital and entrepreneurial orientation of the firm: bonding and bridging effects. Journal of Management, 41(7), 1957-1981. https://doi. org/10.1177/0149206312469666

Caraça, J., Lundvall, B.-Å., \& Mendonça, S. (2009). The changing role of science in the innovation process: From Queen to Cinderella? Technological Forecasting and Social Change, 76(6), 861-867. https: / / doi.org/10.1016/j.techfore.2008.08.003

Chin, R. (1961). The utility of system models and developmental models for practitioners. In Bennis WG, Benne KD, \& Chin R (Eds.), The Planning of Change: Readings in the Applied Behavioral Sciences . New York: Rinehart and Winston.

Clark, T. N. (1968). Institutionalization of innovations in higher education: four models. Administrative Science Quarterly, 13(1), 1-25. https: / / doi.org/10.2307/2391259

Congreso de la República de Colombia. (1990). Ley 29. Recuperado de http:/ / www.alcaldiabogota. gov.co / sisjur / normas / Norma1.jsp? $\mathrm{i}=254$

Congreso de la República de Colombia. (2009). Ley 1286. Recuperado de http://www. alcaldiabogota.gov.co / sisjur/normas / Norma1.jsp?i=34850

Consejo Privado de Competitividad. (2016). Informe nacional de competitividad 2016-2017. Recuperado de http:/ / compite.com.co/wp-content / uploads/2016/11/CPC_Libro_Web_2016-2017. pdf
Coombs, R., Saviotti, P., \& Walsh, V. (1987). Economics and Technological Change. Hong Kong: Rowman \& Littlefield.

Cooper, R. G. (1983). The new product process: an empirically-based classification scheme. RED Management, 13(1), 1-13. https:// doi. org/10.1111/j.1467-9310.1983.tb01124.x

Corbett, A., Covin, J. G., O'Connor, G. C., \& Tucci, C. L. (2013). Corporate Entrepreneurship: state-ofthe-art research and a future research agenda. Journal of Product Innovation Management, 30(5), 812-820. https: / / doi.org/10.1111/jpim.12031

Covin, J. G., \& Slevin, D. P. (1991). A conceptual model of entrepreneurship as firm behavior. Entrepreneurship Theory \& Practice, 16(3), 7-25. https: / / doi.org/1042-2587-91-161

Crane, D. (1972). Invisible Colleges: Diffusion of Knowledge in Scientific Communities. Chicago: University of Chicago Press.

DANE. (2014). Encuesta de Desarrollo e Innovación Tecnológica en el sector Servicios y Comercio EDITS 2012-2013. Recuperado de http: / / www. dane.gov.co / files / investigaciones / boletines / edit/Pres_EDIT_servicios_2012_2013.pdf

DANE. (2015). Encuesta de desarrollo e innovación tecnológica - EDIT (industria manufacturera 2013-2014). Recuperado de http: / / www.dane. gov.co / files / investigaciones / boletines / edit / presentacion_EDIT_manufacturera_2013_2014. pdf

DANE. (2017). Gran Encuesta Integrada de Hogares -GEIH- Mercado Laboral. Bogotá. Recuperado de http://www.dane.gov.co/index.php/ estadisticas-por-tema / mercado-laboral / empleo-y-desempleo

DNP, Observatorio colombiano de ciencia y tecnología, \& C230 Consultores. (2015). Indice Departamental de Innovación para Colombia (IDIC), 2015. Bogotá. Recuperado 
de https: / / colaboracion.dnp.gov.co/CDT / Prensa/Publicaciones/Índice de Innovación Departamental para Colombia.pdf

Durieux, V., \& Gevenois, P. A. (2010). Bibliometric indicators: quality measurements of scientific publication. Radiology, 255(2), 342-351. https: / / doi.org/10.1148/ radiol.09090626

Endeavor Colombia. (2016). A los paisas les falta más ambición para emprender: Endeavor Colombia. Medellín. Recuperado de http:// www.elcolombiano.com/negocios/gec-2016endeavor-y-su-vision-del-emprendimientopaisa-FY3760263

Englander, K. (2009). El mundo globalizado de las publicaciones científicas en inglés: un enfoque analítico para comprender a los científicos multilingües. Discurso \& Sociedad, 3(1), 90-118. https: / / doi.org/1887-4606

Escobar-Sierra, M., Lara-Valencia, L. A., \& ValenciaDeLara, P. (2017). Model for innovation management by companies based on corporate entrepreneurship. Problems and Perspectives in Management, 15(3), 234-241. https://doi. org/http: / / dx.doi.org/10.21511/ppm.15(31).2017.07

Escobar-Sierra, M., \& Vera-Acevedo, L. D. (2016). Inn-Cumbe: un modelo para la gestión de la innovación socialmente responsable, propuesto a partir de un estudio de caso a nivel organizacional. Espacios, 37(17), 5. https: / / doi. org/0798 1015

Fang, Q. (2013). Corporate entrepreneurship: constructs and research focuses. In E. Qi, J. Shen, \& R. Dou (Eds.), The 19th international conference on industrial engineering and engineering management (pp. 699-706). Berlin, Heidelberg: Springer Berlin Heidelberg. https://doi. org/10.1007/978-3-642-38442-4

Fedesarrollo. (2014). Innovación y emprendimiento en Colombia: balance, perspectivas y recomendaciones de política, 2014-2018. (H. J. Gómez \& D.
Mitchell, Eds.), Cuadernos de Fedesarrollo 111 número cincuenta (Vol. 50). Recuperado de http: / / www.repository.fedesarrollo.org.co/ bitstream/handle/11445/149/CDF_No_50_ Marzo_2014.pdf?sequence $=3 \&$ isAllowed $=\mathrm{y}$

Forrest, J. F. (1991). Practitioners' forum, Models of the process technological innovation. Technology Analysis \& Strategic Management, 3(4), 439-453. https: / / doi.org/10.1080/09537329108524070

Freeman, C. (1991). Networks of innovators: a synthesis of research issues. Research Policy, 20(5), 499-514. https://doi.org/ doi:10.1016/0048-7333(91)90072-X

Freeman, C. (1996). The greening of technology and models of innovation. Technological Forecasting and Social Change, 53(1), 27-39. https://doi. org/10.1016/0040-1625(96)00060-1

Freeman, J., \& Engel, J. S. (2007). Models of innovation: startups and mature corporations. California Management Review, 50(1), 94-119. https: / / doi.org/10.2307/ 41166418

Friederichsen, R., Minh, T. T., Neef, A., \& Hoffmann, V. (2013). Adapting the innovation systems approach to agricultural development in Vietnam: challenges to the public extension service. Agriculture and Human Values, 30(4), 555-568. https:/ / doi.org/10.1007/ s10460-0139433-y

GEM. (2014). Dinámica empresarial colombiana 2013. (R. Varela, J. A. Moreno, M. Bedoya, L. Gómez, J. G. Restrepo, I. Negrearte, A. M. Fierro, Eds.). Recuperado de http://gemcolombia.org/ wp-content/ uploads / dinámica-empresarialcolombiana.pdf

Gobierno Nacional, \& FARC-EP. (2016). Acuerdo final para la terminación del conflicto y la construcción de una paz estable y duradera. 248-16, 296.

Godin, B. (2015). Models of innovation: why models of innovation are models, or what work is being done in calling them models? Social 
Nuevo MOdelo DE INNOVACIÓN PARA EL CONTEXTO ORGANIZACIONAL: UNA

RELACIÓN ENTRE EL EMPRENDIMIENTO CORPORATIVO Y LAS PARTES INTERESADAS
Studies of Science, 45(4)(4), 570-596. https:// doi.org/10.1177/0306312715596852

Gokhberg, L., \& Kuznetsova, T. (2011). S\&T and Innovation in Russia: Key Challenges of the Post-Crisis Period. Journal of East-West Business, 17(2-3), 73-89. https: / / doi.org/10.1080/10669 868.2011 .634769

Gómez, L., Martínez, J., \& Arzuza, M. (2006). Política pública y creación de empresas en Colombia. Pensamiento \& Gestión, (21), 1-25.

Guth, W., \& Ginsberg, A. (1990). Guest editors' introduction: Corporate entrepreneurship. Strategic Management Journal, 11, 5-15. https:/ / doi.org/10.1016/j.ymeth.2009.12.016

Hargrave, T. J., \& Van De, A. H. (2006). A collective action model of institutional innovation. Academy of Management Review, 31(4), 864-888. https: / / doi.org/10.5465/AMR.2006.22527458

Hashimoto, M., \& Nassif, V. M. J. (2014). Inhibition and encouragement of entrepreneurial behavior: antecedents analysis from managers' perspectives. BAR - Brazilian Administration Review, 11(4), 385-406. https://doi. org/10.1590/1807-7692bar2014130008

Havelock, R. G. (1970). A Guide to Innovation in Education. Ann Arbor: Center for Research on Utilization of Scientific Knowledge, Institute for Social Research, University of Michigan.

Hennala, L., Parjanen, S., \& Uotila, T. (2011). Challenges of multi-actor involvement in the public sector front-end innovation processes. European Journal of Innovation Management, 14(3), 364 . https://doi. org / 10.1108/14601061111148843

Hobday, M. (2005). Firm-level innovation models: perspectives on research in developed and developing countries. Technology Analysis \& Strategic Management, 17(2), 121-146. https:/ / doi.org/10.1080/ 09537320500088666

Hobday, M., Boddington, A., \& Grantham, A. (2012). Policies for design and policies Vol. 10 • Núm.19 / jul-dic / 2018 for innovation: Contrasting perspectives and remaining challenges. Technovation, 32(5), 272-281. https://doi.org/10.1016/j. technovation.2011.12.002

Kelly, P., \& Kranzberg, M. (Eds.). (1978). Technological Innovation: A Critical Review of Current Knowledge. San Francisco: San Francisco Press.

Kline, S. J., \& Rosenberg, N. (1986). An overview of innovation. In Ralph Landau \& Nathan Rosenberg (Eds.). The Positive Sum Strategy: Harnessing Technology for Economic Growth (pp. 275-305). Washington: National Academy Press.

Kuratko, D. F. (2010). Corporate entrepreneurship: an introduction and research review. In $\mathrm{Z}$. J. Acs \& D. B. Audretsch (Eds.), Handbook of Entrepreneurship Research (pp. 129-163). New York, NY: Springer New York. https://doi. org/10.1007/978-1-4419-1191-9

Langrish, J., Gibbons, M., Evans, W., \& Jevons, F. . (1972). Wealth from knowledge: studies of innovation in industry. London: The Macmillan Press.

López, G. A. (2004). Aproximación a las generalidades y debilidades del sistema de innovación colombiano. Scientia Et Technica (Vol. X). Universidad Tecnológica de Pereira. Retrieved from http:/ / www.redalyc.org/html/849/84912053006/

Lundvall, B.-Å. (Ed.). (2010). National systems of innovation: Towards a theory of innovation and interactive learning (1st ed.). Anthem press.

Marinova, D., \& Phillimore, J. (2003). Models of innovation. In L. V. Shavinina (Ed.), The international handbook on innovation (pp. 44-53). Oxford: Elsevier.

Mead, D. (2016). Innovar es darle sentido humano a las empresas. Medellín. Retrieved from http:/ / www.elcolombiano.com/negocios/innovares-darle-sentido-humano-a-las-empresasAF5412552

Migración Colombia. (2017). El 2\% de los ciudadanos venezolanos que entra a Colombia se queda. Bogotá. 
Recuperado de http://www.portafolio. co/economia / llegada-de-venezolanos-acolombia-508775

Monroy Varela, S. E. (2006). Nuevas políticas y estrategias de articulación del sistema de ciencia, tecnología e innovación colombiano. INNOVAR. Revista de Ciencias Administrativas y Sociales, 16(28), 157-172. Recuperado de http://www.redalyc.org/ $\mathrm{html} / 818 / 81802810 /$

Morris, M., Kuratko, D., \& Covin, J. (2010). Corporate entrepreneurship \& innovation. (M. S. Acuna, Ed.) (3rd ed.). Mason, Ohio: Cengage Learning.

Mulkay, M. J. (1975). Three models of scientific development. The Sociological Review, 23(3), 509-526. https: / / doi.org/10.1111/j.1467954X.1975.tb02231.x

Nelson, R. R. (Ed.). (1993). National innovation systems: a comparative analysis. New York: Oxford University Press, USA.

Nelson, R. R. (2000). National innovation systems and Instituted Processes. In Z. J. Ács (Ed.), Regional innovation, knowledge, and global change (pp. 11-26). Pinter.

Newby, H. (1992). One society, one Wissenschaft: A 21st Century vision. Science and Public Policy, 19(1), 7-14. https:/ / doi.org/10.1093/ spp/19.1.7

OCDE. (2014). Estudios de la OCDE de las Politicas de Innovación: Colombia. Retrieved from https://www.oecd.org/sti/inno/ colombia-innovation-review-assessmentand-recommendations-spanish.pdf

Pinch, T. J., \& Bijker, W. E. (1984). The Social Construction of Facts and Artefacts: Or How the Sociology of Science and the Sociology of Technology Might Benefit Each Other. Social Studies of Science, 14(3), 399-441.

Quesada-Vázquez, J., \& Rodríguez-Cohard, J. C. (2015). Implementation challenges in cluster policy making: the case of the Andalusian Furniture Technology Centre. Prometheus, 33(2), 113-137. https: / / doi.org/10.1080/08109028.20 15.1095976

Quintero-Campos, L. J. (2010). Aportes teóricos para el estudio de un sistema de innovación. INNOVAR. Revista de Ciencias Administrativas y Sociales, 20(38), 57-76.

Rammer, C. (2006). Innovation in firms. In U. Schmoch, C. Rammer, \& H. Legler (Eds.), National Systems of Innovation in Comparison, structure and Performance Indicators for Knowledge Societies (pp. 107-132). Dordrecht: Springer Netherlands. https:/ / doi.org/10.1007/1-40204949-1_7

Real Academia Española. (2017). Diccionario de la lengua española. Recuperado de http:/ / dle.rae. es / ?id=Fy2OT7b

Robertson, T. S. (1971). Innovative behavior and communication. New York: Holt, Rinehart and Winston.

Robledo, J. (2007). De los grupos consolidados de investigación a los sistemas dinámicos de innovación: el desafío actual del desarrollo científico y tecnológico colombiano. Dyna, 74(152), 1-7.

Romo Jiménez, A. M., Valencia-De-Lara, P., \& Escobar-Sierra, M. (2017). Un análisis bibliométrico sobre responsabilidad social empresarial y consumidores. In Diversidad y complejidad organizacional en América Latina. Perspectivas de Análisis (Primera, pp. 181-207). México: Red Mexicana de Investigadores en Estudios Organizacionales (REMINEO) A.C.

Rothwell, R. (1992). Successful industrial innovation: critical factors for the 1990s. RED Management, 22(1992), 221-240.

Saren, M. A. (1984). A classification and review of process models of innovation. RED Management, 14(1), 11-24. https://doi. org/10.1111/j.1467-9310.1984.tb00504.x 
Nuevo MOdelo DE INNOVACión PARA EL CONTEXTO ORGANIZACIONAL: UNA

RELACIÓN ENTRE EL EMPRENDIMIENTO CORPORATIVO Y LAS PARTES INTERESADAS

Schmidt-Tiedemann, K. J. (1982). A Descriptive Model of the Innovation Process. Research Management, 25(2).

Schon, D. A. (1967). Technology and change: the impact of invention and innovation on American social and economic development. New York: Delta Books.

Tait, J., \& Williams, R. (1999). Policy approaches to research and development: foresight, framework and competitiveness. Science and Public Policy, 26(2), 101-112. https://doi. org / 10.3152/147154399781782536

The Global Innovation Index. (2017). I: Country economy profiles: Colombia GII 2017 Report I. recuperado de https://www. globalinnovationindex.org/gii-2017-report\#

Tornatzky, L. G., Eveland, J., Hoylan, M. G., Hetzner, W. A., Johnson, E. C., Roitman, D., \& Schneider, J. (1983). The process of technological innovation reviewing the literature. Washington: National Science Foundation.

Valencia-De-Lara, P., \& Patlán Peréz, J. (2011). El Empresario descubridor de Oportunidades de Mercado: Puntos Centrales de la Teoria de Kirzner. Tec Empresarial, 5(1), 31-38.

Valencia-De-Lara, P., \& Patlán Peréz, J. (2011). El empresario innovador y su relación con el desarrollo económico. Tec Empresaria, 5(3), 21-27.
Van de Ven, A. H. (1989). Research on the management of innovation. (A. H. Van de Ven, H. L. Angle, \& M. S. Poole, Eds.). New York: Oxford University Press.

Van de Ven, A. H., Polley, D., Garud, R., \& Venkataraman, S. (2008). The Innovation Journey. New York: Oxford University Press.

Von Hippel, E. (1978). A customer active paradigm for industrial product idea generation. In M. J. Baker (Ed.), Industrial Innovation (Vol. 7, pp. 240266). London: Palgrave Macmillan. https: / doi. org/10.1007/978-1-349-03822-0_5

Wojan, T. R. (2017). Opportunities and Challenges of Spatially Distributed Innovation Imaginariums (pp. 3-20). Springer, Cham. https://doi. org/10.1007/978-3-319-50547-3_1

Zahra, S. A. (1986). A cannonical analysis of corporate entrepreneurship antecedents and impact on performance. Academy of Management Proceedings, (1), 71-75. https: / / doi.org/10.5465/ ambpp.1986.4978718

Zaltman, G., Duncan, R., \& Holbeck, J. (1973). Innovations and Organizations. New York: John Wiley.

Ziman. (1991). A neural net model of innovation. Science and Public Policy, 18(I), 65-75. https:// doi.org/10.1093/spp/18.1.65 